Cahiers de philosophie de l'université de

\title{
Ce je pense par où il fallait finir. Une lecture rousseauiste du cogito?
}

Jean-Christophe Bardout

\section{OpenEdition}

1 Journals

Édition électronique

URL : https://journals.openedition.org/cpuc/693

DOI : $10.4000 /$ cpuc.693

ISSN : 2677-6529

Éditeur

Presses universitaires de Caen

\section{Édition imprimée}

Date de publication : 31 décembre 2013

Pagination : 55-79

ISBN : 978-2-84133-487-2

ISSN : 1282-6545

\section{Référence électronique}

Jean-Christophe Bardout, "Ce je pense par où il fallait finir. Une lecture rousseauiste du cogito ?» Cahiers de philosophie de l'université de Caen [En ligne], 50 | 2013, mis en ligne le 13 juin 2018, consulté le 05 février 2023. URL : http://journals.openedition.org/cpuc/693; DOl : https://doi.org/10.4000/ cpuc. 693

\section{(c) (7) (8)}

Creative Commons - Attribution - Pas d'Utilisation Commerciale 4.0 International - CC BY-NC 4.0 https://creativecommons.org/licenses/by-nc/4.0/ 


\title{
Ce je pense par où il fallait finir. Une lecture rousseauiste du cogito ${ }^{1}$
}

\begin{abstract}
$\grave{A}$ L'instar de l'auteur du Discours de la méthode, Rousseau cite les philosophes avec parcimonie, et fort peu Descartes. Une page célèbre du livre VI des Confessions ${ }^{2}$ nous apprend qu'il le lit en 1737 aux Charmettes, en compagnie des autres principaux philosophes modernes (Arnauld et Nicole avec La logique de Port-Royal, Locke, Malebranche, Leibniz, etc.). Cette énumération ouverte se conclut par le constat du désaccord des philosophes, constat banal en lui-même, mais déterminant dans l'image que Jean-Jacques va se forger de l'histoire de la philosophie ${ }^{3}$. Relevons les principaux lieux du corpus rousseauiste où il est fait mention de Descartes, afin d'esquisser notre hypothèse directrice.
\end{abstract}

\section{Un bilan contrasté}

Du nom de Descartes, nous rencontrons seulement une douzaine de mentions dans le corpus rousseauiste, qui se laissent distribuer en trois groupes.

Parmi elles, une demi-douzaine concerne la physique, avec des allusions généralement négatives à la théorie des tourbillons ${ }^{4}$. C'est le cas dès 1737 , dans

1. La version écrite de ce texte a bénéficié de la relecture attentive et des remarques de L. Verhaeghe. Le texte de la communication prononcée a été relu par G. Olivo. Qu'ils en soient ici remerciés.

2. Les réferences aux œuvres de Rousseau sont données d'après l'édition des Euvres complètes, Bernard Gagnebin et Marcel Raymond (éd.), Paris, Gallimard (Bibliothèque de la Pléiade), 1959-1995, 5 vol., citées OC suivi du tome et de la page; les Lettres morales seront citées $L M$. Ici: OC I, 237. Pour un repérage des principaux lieux cartésiens dans l'œuvre de Rousseau, voir A. Charrak, "Descartes», in Dictionnaire de Jean-Jacques Rousseau, R. Trousson et F. Eigeldinger (dir.), Paris, H. Champion, 2006, p. 204-206.

3. Cette histoire se présente comme l'affrontement sans fin des différents systèmes philosophiques. Le tableau qu'en dresse Rousseau dans LM II (in OC IV, 1089) est classique, mais emprunte peut-être certains éléments à la tradition cartésienne ainsi qu’à Pascal, comme l'usage critique du concept de secte philosophique.

4. Voir ainsi J.-J. Rousseau, Du contrat social, liv. II, chap. IX, in OC III, 388. 
un passage du Verger de madame de Warens, qui renvoie probablement à la période dont la page des Confessions évoquée plus haut se fera plus tard l'écho:

Tantôt avec Leibnitz, Mallebranche, et Newton

Je monte ma raison sur un sublime ton,

J'examine les Loix des corps et des pensées:

Avec Locke, je fais l'histoire des idées [...]

Tantôt à la Physique appliquant mes problémes,

Je me laisse entraîner à l'esprit de sistémes;

Je tâtonne Descarte et ses égarements

Sublimes, il est vrai, mais frivoles Romans 5 .

Ce premier texte appelle deux brèves remarques. En premier lieu, la relégation de la physique cartésienne au rang de roman est banale à l'époque, notamment depuis la $13^{\mathrm{e}}$ Lettre philosophique de Voltaire en $1734^{6}$. En outre, Descartes est pour ainsi dire réduit à sa physique: c'est un faiseur de systèmes et d'hypothèses, disqualifié en physique, mais peut-être aussi en métaphysique ${ }^{7}$.

Dès le Discours sur les sciences et les arts de 1750, la critique des sciences en passe par un développement sur la difficulté à exhiber un critérium de la vérité. En un raccourci frappant, Rousseau renvoie dos à dos Descartes et la philosophie péripatéticienne: Descartes n’a-t-il pas élaboré une hypothèse insoutenable pour expliquer le système de la nature, plutôt que d'avouer son ignorance ${ }^{8}$ ?

5. OC II, 1128.

6. Parlant de Locke, Voltaire écrit: «Tant de raisonneurs ayant fait le roman de l'âme, un sage est venu qui en a fait modestement l'histoire», Lettres philosophiques, R. Naves (éd.), Paris, Garnier, 1962, Lettre XIII, p. 63; voir Lettre XIV, p. 75; «Qu’on fasse son éloge, à la bonne heure, pourvu qu'on ne fasse pas celui de ses romans philosophiques, méprisés aujourd'hui pour jamais dans toute l'Europe», Le philosophe ignorant, in Euvres complètes, L. Moland (éd.), Paris, Garnier, 1877-1885, t. XXVI, chap. V, p. 50; «Le philosophe, en rendant hommage au génie de Descartes, foule aux pieds les ruines de ses systèmes ", Dictionnaire philosophique, in CEuvres complètes, t. XVIII, art. «Cartésianisme», p. 6o.

7. L'examen critique de la troisième Lettre morale place, en apparence du moins, physique et métaphysique sur le même rang d'imperfection et d'inachèvement: «La simple phisique ne nous est pas moins obscure que la métaphysique et la morale», OC IV, 1096. Si le vocabulaire dont use la $L M$ III (en parlant de «puerilités» et de «réveries», OC IV, 1095) peut conduire à considérer que la critique vise d'abord la physique cartésienne, c'est cependant toute la philosophie (donc aussi la métaphysique) qui est finalement mise en cause. Après l'avènement de l'empirisme lockien et de la physique newtonienne, "Adieu toute la philosophie du sage et methodique Descartes", ibid., 1096, nous soulignons. On va voir que, si le rejet de la physique est complet, la réserve envers la métaphysique (en ses résultats positifs) n'atteint cependant pas son premier principe.

8. «Moins on sait, plus on croit savoir. Les péripatéticiens doutoient-ils de rien? Descartes n'a-t-il pas construit l'univers avec des cubes et des tourbillons?", OC III, 18. À ces textes, il conviendrait d'ajouter, comme le suggère L. Verhaeghe dans un article à paraitre 
Une seconde série de mentions fait de Descartes une sorte de héros de la raison et de la philosophie, indépendamment du contenu effectif de la sienne ${ }^{9}$. Le premier Discours contient en effet une autre mention de Descartes, plus intéressante, qui tempère l'appréciation négative qu'on vient d'évoquer: cette fois, Descartes est mentionné en compagnie de ces deux autres maîtres incontestés de la physique moderne que sont Bacon et Newton ${ }^{10}$. Descartes n'est plus stigmatisé comme un faiseur de romans, mais loué comme un précepteur du genre humain.

$\mathrm{Si}$, pour Voltaire, la physique newtonienne a définitivement supplanté les romans cartésiens, Rousseau interprète différemment la succession des systèmes d'explication de la nature; cette succession même les frappe tour à tour de caducité, et incite à la plus grande modération dans l'appréciation critique: l'électricité sera ainsi à Newton ce que l'attraction fut à Descartes ${ }^{11}$. Faut-il dès lors considérer que Rousseau se contredit en érigeant un faiseur de roman au rang de précepteur des hommes? Ne convient-il pas plutôt d'entendre que Descartes est un précepteur du genre humain en un autre sens que Bacon et Newton ${ }^{12}$ ?

(L. Verhaeghe, «La troisième Lettre morale ou Rousseau critique pascalien de Descartes», Les Études philosophiques), l'examen plus détaillé de la philosophie cartésienne auquel se livre la troisième $L M$, in OC IV, 1095-1096. Si la physique cartésienne y est incontestablement mise en cause, la métaphysique de Descartes y est également visée, à l'exception (nous y revenons longuement) de son premier principe.

9. Voir notamment la dernière réponse consécutive au premier Discours, in $O C$ III, 92, où Descartes se trouve présenté comme un exemple d'abnégation et de désintéressement, au regard des professions lucratives. À la fin de la deuxième $L M$, in OC IV, 1091, Descartes apparait, brièvement et de manière quelque peu convenue, comme une sorte de champion de la raison: «Que fera donc celui qui cherche sincérement la vérité parmi ces foules de savans qui tous prétendent l'avoir trouvée et se démentent mutuellement? Pesera-t-il tous les sistêmes? Feuillettera-t-il tous les livres, ecoutera-t-il tous les Philosophes, comparera-t-il toutes les sectes, osera-t-il prononcer entre Épicure et Zénon [...] Osera-t-il préférer ses lumiéres à celles de Pascal et sa raison à celle de Descartes?». On relève le jugement plutôt laudateur à l'endroit de Pascal, nous y reviendrons. On relève aussi toute l'ambiguïté du statut de Descartes; celui-ci est loué pour l'ampleur et la qualité de sa «raison»; mais, si l'on veut être cartésien jusqu'au bout, ne vaut-il pas mieux préférer sa raison bien conduite à celle de Descartes lui-même?

10. «Il n'a point fallu de maitres à ceux que la nature destinoit à faire des disciples. Les Verulams, les Descartes et les Newtons, ces Precepteurs du Genre-humain n'en ont point eu euxmêmes, et quels guides les eussent conduits jusqu'où leur vaste genie les a portés? », Du contrat social, in OC III, 29.

11. $L M$ III, in OC IV, 1096.

12. Relevons dès maintenant que, dans le texte précité du premier Discours, ce qui élève un auteur au rang de précepteur n'est pas précisé. Si Newton en fut un en physique, rien n'indique que Descartes le fut pour des motifs similaires. Le précepteur se caractérise en effet par sa liberté à l'égard de toute filiation intellectuelle, et sa capacité à faire école, parce qu'il infléchit de manière décisive le développement de sa discipline, en imposant une manière nouvelle de formuler les problèmes. 
Cette critique de la physique cartésienne (mais tout aussi bien newtonienne) reste une constante de la pensée rousseauiste, jusque dans la Profession de foi du vicaire savoyard ${ }^{13}$. Rousseau s'en prend alors à une épistémologie de la loi, qui prétend ignorer les causes réelles des phénomènes observables, au profit d'une pure reconstitution hypothétique ${ }^{14}$.

Selon un topos fréquent dans la réception du cartésianisme depuis la fin du XVII ${ }^{\mathrm{e}}$ siècle, Descartes ne vaut plus par ce qu'il a écrit, mais d'abord en raison de ce que sa méthode a rendu possible, fût-ce à son propre encontre ${ }^{15}$.

13. «S'il faut admettre des loix générales dont je n’apperçois point les rapports essentiels avec la matiére, de quoi serai-je avancé? Ces loix, n'étant point des êtres réels, des substances, ont donc quelqu'autre fondement qui m'est inconnu. L'expérience et l'observation nous ont fait connoitre les loix du mouvement, ces loix déterminent les effets sans montrer les causes; elles ne suffisent point pour expliquer le sistême du monde et la marche de l'univers. Descartes avec des dés formoit le ciel et la terre, mais il ne put donner le prémier branle à ces dés, ni mettre en jeu sa force centrifuge qu'à l'aide d'un mouvement de rotation. Newton a trouvé la loi de l'attraction; mais l'attraction seule réduiroit bientot l'univers en une masse immobile; à cette loi il a falu joindre une force projectile pour faire décrire des courbes aux corps célestes. Que Descartes nous dise quelle loi physique a fait tourner ses tourbillons; que Newton nous montre la main qui lança les planetes sur la tangente de leurs orbites», Émile, liv. IV, in OC IV, 575-576.

14. Il vise donc probablement et entre autres les articles 45 à 47 de la troisième partie des Principia philosophice de Descartes.

15. Pour bon nombre d'auteurs, on peut en appeler à Descartes, non pour le suivre, mais pour le corriger ou le contredire, au nom d'une liberté de philosopher et d'une exigence de rationalité dont il a lui-même donné l'exemple le plus éclatant. Voir B. de Fontenelle, Poésies Pastorales. Avec un Traité sur la Nature de l'Eglogue, et une Digression sur les Anciens et les Modernes, Paris, M. Guérout, 1688, p. 248: «C'est luy [Descartes] [...] qui a amené cette nouvelle methode de raisonner, beaucoup plus estimable que sa Philosophie mesme, dont une bonne partie se trouve fausse ou fort incertaine, selon les propres regles qu'il nous a apprises» ou édition des Euvres complètes de Fontenelle, A. Niderst (éd.), Paris, Fayard (Corpus des œuvres de philosophie en langue française), 1989-2001, t. II, p. 420, texte recopié sans référence dans l'article «Logique» de l'Encyclopédie (t. IX, p. 638); voir aussi l'article "Cartésianisme», dû à l'abbé Pestré, t. II, p. 725. Le thème est fréquent au XVIII siècle; voir $\mathrm{C}$. Buffier, Traité des premières véritez et de la source de nos jugements, Paris, $V^{\text {ve }}$ Mongé, 1724, $\$ 5$, p. 4-5 ou le même extrait dans C. Buffier, Euvres philosophiques, F. Bouillier (éd.), Paris, 1843, p. 4; «les armes dont nous nous servons pour le combattre ne lui en appartiennent pas moins, parce que nous les tournons contre lui», D'Alembert, Discours préliminaire de l'Encyclopédie; "C'est suivre l'esprit de ce grand homme que d'abandonner ses idées lorsqu'elles se trouvent peu solides», J.-P. de Bougainville, «Discours préliminaire à la traduction de l'Anti-Lucrèce», in M. de Polignac, L'Anti-Lucrèce, Paris, 1749, p. LIIJ; «Descartes a enseigné à ceux qui sont venus après lui, à découvrir ses erreurs mêmes", Montesquieu, Mes pensées, $\mathrm{n}^{\circ}$ 775, p. 1074. Au moment où il proclame son désaccord avec Descartes, Charles Bonnet voit encore dans «le grand Descartes» «le célebre Inventeur de la Méthode de philosopher ", La palingénésie philosophique, Genève, C. Philibert et B. Chirol, 1769, t. I, part. XI, p. 419. Voir E. Bonnot de Condillac, Essai sur l'origine des connaissances humaines, ${ }^{\mathrm{e}}$ partie, sect. II, ch. III, $\$ 41$, A. Bertrand (éd.), Paris, Vrin (Bibliothèque des textes philosophiques), 2002, p. 185. 
Le bilan de notre inventaire semble donc peu éloquent, et la moisson bien maigre. Des thèses cardinales de la métaphysique, et du cogito en particulier, on ne trouve jusqu'ici aucune mention significative.

Explorons cependant une dernière série de mentions de Descartes, rassemblées dans les deux textes qui vont nous retenir, les Lettres morales de 1757 (singulièrement la troisième) et la Profession de foi du vicaire savoyard. L'exposé autobiographique préparatoire à la profession de foi proprement dite rapproche l'état de doute et d'incertitude qu'éprouve le vicaire avant de tenter de fixer ses opinions, des dispositions exigées par Descartes pour la recherche de la vérité ${ }^{16}$. Cette fois, nous sommes bien en présence d'une allusion directe à la métaphysique cartésienne, et plus spécialement au doute qui inaugure ses différents exposés, du Discours de la méthode aux Principes de la philosophie. Ce rapprochement avec Descartes, initié par Rousseau lui-même, appelle toutefois une remarque, qui nous permettra de formuler notre hypothèse, puis d'énoncer deux réquisits de l'enquête.

Comme l'a bien vu Henri Gouhier, dans le chapitre II des Méditations métaphysiques de Jean-Jacques Rousseau ${ }^{17}$, les doutes du vicaire et la crise dont ils témoignent sont de nature morale et religieuse: ils ne sont pas faits pour durer, et ne cherchent pas à mettre en évidence un éventuel premier principe certain et indubitable. En d'autres termes, le doute du vicaire s'impose à lui comme de l'extérieur, et n'a rien de méthodique. Il est donc, d'emblée, d'une autre nature, et vise d'autres fins que le doute cartésien. Si le doute rousseauiste tend, lui aussi, à sa propre suppression parce qu'il représente un état violent et inquiétant ${ }^{18}$, il ne constitue pas la condition de possibilité d'une première certitude contre laquelle il vient se briser. Autrement dit, à supposer qu'il y ait un cogito rousseauiste, celui-ci, dans la Profession du moins ${ }^{19}$, ne s'ensuivra pas d'un doute méthodique et

16. «J'étois dans ces dispositions d'incertitude et de doute que Descartes éxige pour la recherche de la vérité. Cet état est peu fait pour durer, il est inquiétant et pénible, il n'y a que l'intérest du vice ou la paresse de l'ame qui nous y laisse», Émile, liv. IV, in OC IV, 567.

17. H. Gouhier, Les méditations métaphysiques de Jean-Jacques Rousseau, $2^{\mathrm{e}}$ éd., Paris, Vrin (Bibliothèque d'histoire de la philosophie), 1984. L'historiographie a depuis longtemps relevé des proximités entre le cartésianisme et la Profession de foi. Voir F. Bouillier, Histoire de la philosophie cartésienne, $3^{\mathrm{e}}$ éd., Paris, Delagrave, 1868, t. II, ch. XXXII, p. 632.

18. "Le doute sur les choses qu'il nous importe de connoitre est un état trop violent pour l'esprit humain ", Émile, liv. IV, in OC IV, 568. Voir J.-J. Rousseau, Lettres philosophiques, H. Gouhier (éd.), Paris, Vrin (Bibliothèque des textes philosophiques), 1974, «Lettre à Voltaire du 18 août 1756 ", p. 43 ou OC IV, 1070

19. L. Verhaeghe, dans l'article précité, suggère qu'il n'en va pas de même dans la $L M$ III, qui serait le seul lieu rousseauiste d'un doute méthodique, mais surtout volontaire et conduit audelà de ses bornes naturelles. $\mathrm{Si}$, en ce sens, les $L M$ marquent une plus grande proximité avec Descartes, nous verrons que, par son effort pour tenter de «déduire» des vérités du premier principe incontestable, c'est bien l'exposé du vicaire qui marque cette proximité plus grande. 
hyperbolique. Le refus de s'inscrire dans une filiation cartésienne se confirme dans les pages suivantes de la Profession, où Descartes n'apparaît plus (explicitement du moins) que pour être rejeté, notamment en physique ${ }^{20}$.

Nous pouvons dès lors énoncer notre hypothèse : il est loisible de mettre en évidence un cogito de Rousseau, qui se formule dans une confrontation paradoxale mais explicite avec le cartésianisme. Par confrontation, on n'entendra pas ici une fin de non-recevoir ou une récusation manifeste, et pas davantage une répétition explicite ou implicite de Descartes, une sorte d' «à la manière de», tel qu'il a été mis en évidence par Henri Gouhier, à propos d'autres points de tangence entre la pensée de Jean-Jacques et le cartésianisme ${ }^{21}$. Par confrontation, on désigne ici une lecture critique. Dans le cas du cogito, qui seul nous occupera ici, cette confrontation à Descartes prend la forme de ce qu'on peut nommer une réécriture, ou une transposition affective du «je pense». Cette transposition privilégie le dernier mode de la pensée recensé par Descartes dans la liste qu'il en donne à plusieurs reprises, à savoir le sentir ${ }^{22}$. Il reste précisément à déterminer si la transposition fait entendre autrement la même musique, ou donne finalement naissance à une nouvelle partition. Il semble plus que probable que cette transposition et l'inversion de l'ordre de priorité des modes du «je pense» induisent un profond bouleversement dans l'usage que la philosophie peut faire du cogito ${ }^{23}$.

L'hypothèse d'une réécriture rousseauiste du cogito recèle au moins deux enjeux, de nature différente. Historiquement, on peut tout d'abord demander ce qu'une éventuelle répétition rousseauiste du cogito nous apprend à son sujet. Autrement dit, à supposer qu'elle existe effectivement, la réécriture de Jean-Jacques déploie-t-elle une authentique virtualité de l'énoncé cartésien, qui nous donne d'en approfondir le sens? En second lieu, en déclarant qu’on peut finir par le «je pense», Rousseau ne donne-t-il pas à comprendre que le cogito n'est pas seulement un principe, premier maillon d'une chaîne de raisons, mais détermine radicalement le mode de notre relation à l'étant? Il est notable, à cet égard, que les deux textes où se déploient l'examen du cartésianisme et la réécriture du «je pense» sont aussi

20. Voir OC IV, 575-576, cité supra.

21. On songe à la théorie du jugement, ou à la «psychologie de l'erreur». Voir H. Gouhier, Les méditations métaphysiques...

22. Le sentir est dernier dans la liste que produit la «Méditation seconde»; il devient toutefois premier dans l'art. 32 des Principes (AT IX-2, 39) : «Ainsi sentir, imaginer, et mesmes concevoir des choses purement intelligibles, ne sont que des façons differentes d'appercevoir».

23. Au regard de son statut cartésien, une des inflexions les plus notables tient au fait que le «je pense» de Jean-Jacques inaugure une chaîne de raisons, du moins de vérités bien différentes de l'itinéraire cartésien. 
les principaux lieux où Rousseau se confronte à la tradition métaphysique, et plus encore à ses questions les plus fondamentales ${ }^{24}$.

Cette confrontation se déploie en deux lieux textuels principaux, qui sont cependant loin d'être convergents: la troisième Lettre morale tout d'abord, qui constitue l'unique développement dans lequel Rousseau conduit un examen quelque peu suivi de la métaphysique cartésienne, et spécialement de son moment inaugural, avant de conclure par une sorte de retournement du cartésianisme. Il nous faudra en effet comprendre pourquoi Rousseau nous dit, en conclusion du développement dans lequel il examine le savoir des philosophes, qu'il faut finir par où Descartes a commencé, à savoir par le «je pense donc j'existe» ${ }^{25}$.

Nous pourrons alors nous tourner vers le début de la Profession de foi de l'Émile. On y voit Rousseau partir d'une vérité première, «j'existe et j'ai des sens ${ }^{26}$, que nous proposons de lire comme l'énoncé du cogito transposé. De cette assertion initiale, il progresse vers d'autres affirmations, qu'il présente comme des conséquences déduites du j'existe comme être affecté. Cet incipit de l'exposé "métaphysique» du vicaire conduit à se demander pourquoi Jean-Jacques semble ainsi se contredire, en entamant son exposé définitif par où le propos privé des Lettres morales recommandait de finir ${ }^{27}$. La réponse à cette question mettra en évidence la transposition $\mathrm{du}$ «je pense» que nous voulons souligner.

Précisons maintenant les deux réquisits méthodologiques de notre enquête.

Le premier, de nature interprétative, concerne la lettre et la teneur du cogito cartésien. Par ce terme, on entendra, classiquement, l'énoncé par lequel la pensée qui se pense s'assure de sa propre existence comme indubitable. On entendra en outre (et surtout) l'élucidation de la res cogitans conduite par Descartes dans la "Méditation seconde». On le sait, afin d'expliciter la nature de la chose qui pense, Descartes énumère les différents modes qui lui appartiennent, du doute à la sensation ou sentiment ${ }^{28}$. Si l’on réduit le cogito au seul énoncé du «je pense, donc je suis», tel qu'il

24. Si le fait semble assez évident s'agissant de la première partie de l'exposé du vicaire, on se persuadera qu'il en va de même pour la troisième $L M$. Ainsi, après avoir examiné la métaphysique cartésienne, Rousseau déclare: "Où sommes nous? Que voyons nous, que savons nous, qu'est ce qui existe? », OC IV, 1098-1099.

25. OC IV, 1099 .

26. OC IV, 570 .

27. La contradiction n'est qu'apparente, tant les fins des deux textes (surtout si on s'en tient à la $L M$ III sans considérer les suivantes) sont différentes et, pour ainsi dire, sans commune mesure.

28. Voir «Méditation seconde», in Méditations, AT IX-1, 22. 
figure par exemple dans le Discours de la méthode ${ }^{29}$, il n'est, semble-t-il, que partiellement pertinent de parler d'un cogito de Rousseau, ou d'une réception rousseauiste du cogito. On peut en revanche suggérer qu'on est en présence d'une telle réception, à condition d'entendre par cogito: 1) l'énoncé d'une vérité première qui a rang de premier principe; 2) la position de ce principe comme le fondement d'une chaîne de raisons; 3) enfin et principalement le déploiement ou l'explicitation du «je pense", selon une série de modi cogitandi incluant le doute, l'intellection, la volonté, l'imagination et la sensation ${ }^{30}$.

Notre second réquisit, de nature méthodologique, concerne le mode de présence conceptuelle de Descartes dans le corpus rousseauiste. Avant de revenir à sa présence proprement théorique, rappelons que le propos de Rousseau est saturé de réminiscences du lexique et des locutions cartésiens. En outre, Henri Gouhier a bien mis en lumière le recours aux schèmes narratif du Discours de la méthode pour mettre en scène, dans la troisième promenade des Rêveries, le récit de la crise spirituelle et de la réforme intellectuelle et morale qui conduit à la Profession de foi de 1762. De Descartes, Jean-Jacques n'est ni un épigone, ni un adversaire. L'auteur des Méditations métaphysiques n'est ni une source directe ou un horizon immédiat, ni une cible privilégiée (comme les "philosophes», tels Diderot et Helvétius, sans cesse présents à l'esprit du vicaire savoyard) ${ }^{31}$. Il y a donc, au regard du cartésianisme, une distance théorique apparente paradoxalement plus grande que celle qui sépare Rousseau de ses adversaires déclarés, ses contemporains. Ajoutons que la réception rousseauiste de Descartes, textuellement immédiate (au sens où Jean-Jacques a lu directement l'auteur du Discours de la méthode), est cependant médiatisée, et donc filtrée, par plusieurs intermédiaires. Parmi eux, Malebranche tient une place de choix ${ }^{32}$, et l'on pourrait aisément montrer que de nombreux déplacements imposés à des thèses cartésiennes procèdent en fait de réécritures malebranchistes ${ }^{33}$. Un exemple

29. AT VI, 32.

30. "Qu'est-ce qu'une chose qui pense? C'est à dire une chose qui doute, qui conçoit, qui affirme, qui nie, qui veut, qui ne veut pas, qui imagine aussi, et qui sent», "Méditation seconde», in Méditations, AT IX-1, 22; Principia philosophice, Pars prima, $\$ 9$, AT VIII-1, 7; Principes, Première partie, $\$ 9$, AT IX-2, 28 (trad. fr.).

31. La critique des philosophes des Lumières, singulièrement de leur arrogance intellectuelle et de leur culte du progrès matériel, est déjà très présente dans les $L M$, notamment $L M$ II, in $O C \mathrm{IV}, 1088$.

32. Il faudrait probablement faire aussi toute sa place à Pascal (spécialement au fragment 199, «Disproportion de l'homme»), notamment dans l'entreprise de déstabilisation généralisée de toutes les certitudes humaines, comme le suggère Laure Verhaeghe, dans l'article déjà cité.

33. Nous avons illustré, à propos de Kant, cet aspect de la réception du cartésianisme au XVIII ${ }^{e}$ siècle dans notre étude "La conquête de l'existence par la subjectivité", in Descartes en Kant (Actes du Congrès de Paris, 3-5 juin 2004), M. Fichant, J.-L. Marion (dir.), Paris, 
frappant nous en est donné dans la Profession de foi ${ }^{34}$ lorsque Rousseau déclare que nous sentons infailliblement notre âme et sa distinction d'avec notre corps, sans toutefois en connaître l'essence ou la nature par une idée claire et distincte ${ }^{35}$. Il suit de la présence de ces médiations que la lecture rousseauiste de Descartes est sélective: Jean-Jacques prélève, réutilise, transforme ou transpose ${ }^{36}$. L'hypothèse d'une réception du cogito chez Rousseau n'a de sens et d'intérêt que si l'on accepte d'entendre celui-ci comme une parole sur l'essence et la situation du moi, ou, si l'on veut, de la subjectivité, et non comme la répétition d'une thèse de métaphysique cartésienne, dans la fidélité à l'ordre des raisons qui l'impose, et qu'elle commande.

\section{Le je pense, un bon début?}

Nous pouvons à présent ouvrir les Lettres morales. On sait que cet écrit, rédigé à la toute fin de 1757 à l'intention de son amie Sophie d'Houdetot, constitue, en dépit des différences de statut des deux textes, mais aussi de plusieurs divergences, un moment important dans la genèse de la Profession de foi de l'Émile ${ }^{37}$. Ces lettres entendent fournir à leur destinataire des maximes de morale, autrement dit exposer les sentiments de l'auteur sur l'usage de la vie, selon une expression déjà cartésienne ${ }^{38}$. Il convient de

PUF (Épiméthée), 2006, p. 213-238. La fréquentation de Malebranche a été étudiée par Émile Bréhier dans son célèbre article: «Les lectures malebranchistes de Jean-Jacques Rousseau ", Revue internationale de philosophie, $\mathrm{n}^{\circ}$ 1, 1938, p. 98-120.

34. OC IV, 590 .

35. Rousseau abandonne donc la lettre du cartésianisme, mais suit Malebranche, Recherche de la vérité, III, II, ch. VII en particulier. La thèse selon laquelle les sens ne sont pas faits pour nous informer sur la nature des choses, mais sur ce qui est bon pour nous (LM III, in OC IV, 1092-1093) relève encore d'un fond désormais indistinct de cartésianisme et de malebranchisme. On pourrait, de même, à la suite de P.-M. Masson et H. Gouhier, multiplier les rapprochements qui affleurent dans la Profession de foi. Voir ainsi à propos de l'amour de l'ordre, Émile, liv. IV, in OC IV, 593. Dans d'autres cas cependant, Malebranche n'est pas tant lu pour lui-même que comme transmetteur d'analyses pascaliennes; ainsi dans la LM III (OC IV, 1093-1095): l'insistance sur les erreurs de la vue, et le caractère relatif de la perception des grandeurs, avec l'exemple du ciron, démarque certes de près la Recherche de la vérité (I, ch. VI) ; mais l'exemple du ciron précisément, et surtout la défiance envers la raison autant qu'envers les sens, nous éloigne cette fois des intentions malebranchistes et suggèrent fortement l'utilisation d'éléments théoriques issus du fragment 199 de Pascal.

36. Mutatis mutandis, les catégories historiographiques et interprétatives mises en œuvre par Ferdinand Alquié dans Le cartésianisme de Malebranche, Paris, Vrin (Bibliothèque d'histoire de la philosophie), 1974, pour évaluer le cartésianisme d'un Malebranche pourraient être reprises, à condition toutefois de considérer que ce qu'Alquié nommait le "cartésianisme explicite» régresse, pour laisser place à ce qu'il baptisait «cartésianisme transformé» (ibid., ch. VII, p. 299).

37. Elles sont du reste déjà une forme de profession de foi, $L M \mathrm{I}$, in OC IV, 1085.

38. Voir notamment les «Secondes réponses», AT IX-1, 116. 
prêter attention au contexte, et de prendre la mesure de la complexité de la confrontation au cartésianisme. Celle-ci prend place dans l'exposé d'un projet intellectuel clairement non cartésien, lequel se dit néanmoins en termes vaguement cartésiens. Il s'agit en effet d'examiner une fois tout ce qu'on pense ${ }^{39}$, expression qui fait inévitablement songer au semel in vita, de la «Première méditation ${ }^{40}$. Ceci nous conduit très vite à manifester l'inanité presque complète de la philosophie raisonnante, et, contre la raison et le raisonnement, à frayer la voie du sentiment, dont les enseignements seront exploités dans les lettres suivantes. La deuxième lettre étale à l'envi l'impuissance des philosophes dont la diversité trahit le goût de briller, secrètement travaillés qu'ils sont par l'amour-propre. Pour autant, l'hostilité envers les philosophes ne signifie pas l'abandon de la philosophie: «telle est la philosophie dont je cherche à vous instruire», déclare Rousseau à sa correspondante ${ }^{41}$. De quelle philosophie s'agit-il ? C'est précisément Descartes qui va nous permettre de le dire.

Dans notre perspective, la troisième Lettre morale semble gouvernée par un paradoxe. Le cartésianisme a échoué, non pas parce qu'il est une mauvaise philosophie, mais en dépit du fait qu'il est la meilleure, et parce que Descartes fait, en philosophie, exception aux discours aventureux des philosophes. La finalité de la lettre III nous est indiquée à sa fin ${ }^{42}$ : il s'agit de faire des conjectures certes peu probables, mais suffisantes pour établir le doute, et renvoyer dos à dos la multiplicité des discours philosophiques. On y voit même Rousseau défendre provisoirement des positions qui ne sont pas les siennes, et notamment un sensualisme qui lui permet stratégiquement d'ébranler les systèmes, avant de retourner, contre ce même sensualisme ${ }^{43}$, la certitude première du «je pense».

La lettre III progresse selon trois moments.

39. Voir $L M I I$, in OC IV, 1087. Si les commentateurs sont fondés à signaler l'écart final (JeanJacques se propose d'examiner ses opinions pour être heureux autant qu'on peut l'être), la séquence rousseauiste en manifeste un autre: il s'agit certes «d'examiner une fois tout ce qu'on pense» (ce qui appelle un rapprochement cartésien), mais aussi «tout ce qu'on croit, tout ce qu'on sent», ce qui le minore, voire l'interdit, tant il est vrai que «ce qu'on croit» et doit croire (à entendre le mot en sa rigueur) est exclu par Descartes de l'examen (Principia philosophioe, AT VIII-1, 25).

40. AT IX-1, 13.

41. $L M$ II, in OC IV, 1088.

42. OC IV, 1098.

43. "C'est d'eux [les sens] que nous viennent toutes nos idées, ou du moins toutes sont occasionnées par eux ", LM III, in OC IV, 1092; on notera la correction qui tempère le sensualisme par une sorte de vulgate occasionnaliste. Les idées numériques ne sont produites par l'esprit "qu'à l'occasion de mes sensations», Émile, liv. IV, in OC IV, 572 ; voir p. 573. 
Tout d'abord, les premières pages ${ }^{44}$ développent des considérations d'apparence sceptiques et assez convenues, surtout en ce milieu du XVIII ${ }^{e}$ siècle ${ }^{45}$ : nous ne pouvons ainsi saisir l'essence des choses extérieures ou de notre esprit, et il faut reconnaître que nos connaissances sont bornées. Mais il faut ici se méfier des apparences: il ne s'agit pas de réhabiliter le scepticisme, ou de promouvoir un phénoménisme (comme le suggère un temps la métaphore de la maison mal éclairée par des fenêtres trop étroites). On se trouve bien plutôt en présence d'un geste de repli de la connaissance sur ce qui est éprouvé, ou perçu à titre d'affection ${ }^{46}$. Rousseau ne dit pas que nous ne pouvons rien connaitre, mais que nos lumières portent comme nos mains ${ }^{47}$. Nous ne connaissons que le proche, à la lettre, que ce qui nous touche. Ce repli sur la sphère de l'immédiat prend corps en une sentence expresse: «Nous sommes de tout point aveugles» ${ }^{48}$. On note que l'expression admet deux sens. On peut comprendre tout d'abord que nous ne connaissons pas notre propre pouvoir de connaître et ses vraies limites. Mais, plus profondément, on peut entendre que le paradigme cognitif du voir, fondé sur l'évidence de la représentation, est disqualifié ${ }^{49}$. Prêtons attention à la précision: nous ne sommes pas des aveugles de rencontre ou par accident; nous le sommes de naissance, c'est-à-dire que la vue est irrémédiablement étrangère à notre relation à ce qui est: «Nous sommes de tout point aveugles, mais aveugles nés qui n'imaginons pas ce que c'est que la vüe $[\ldots] »^{50}$. Rousseau prépare ainsi une autre manière de penser notre rapport à ce qui est, et récuse le régime de l'objectivité, ou si l'on veut, de

44. $L M$ III, in OC IV, 1092-1094

45. L'analyse des erreurs de la vue fait, là encore, songer à la Recherche de la vérité (I, ch. VI), notamment cette déclaration: "nous ne savons pas même ce que c'est que grandeur et petitesse. La grandeur apparente des objets est relative à la stature de celui qui les mesure» (LM III, in OC IV, 1094). De même, l'exemple de l'eau tiède, repris pour souligner la subjectivité des qualités sensibles, rappelle les analyses malebranchistes concernant la relativité des sensations de froid et de chaud (Recherche de la vérité, I, ch.XIII, $\$$ v), voir OC I, 150-151.

46. P. Audi attire l’attention sur la signification profonde de la séquence qui répond au «Nous ne savons rien" (LM III, in OC IV, 1092), dans l'annexe I de son ouvrage (Rousseau, une philosophie de l'âme, Paris, Verdier, 2008), consacrée au cartésianisme de Rousseau, notamment p. 356 sq. À cette affirmation initiale répond, comme en écho, la succession d'interrogations fébriles déjà citée (LM III, in OC IV, 1098-1099).

47. "Nos courtes lumiéres n'atteignent comme nos mains qu’à deux pieds de nous», ibid., p. 1092.

48. Ibid., p. 1092.

49. Ainsi le vicaire, pour décrire l'expérience originairement vérace de l'union de l'âme et du corps, parle d'un sentiment plus fort que toute évidence, et significativement comparable au sentiment de l'existence lui-même, Émile, liv. IV, in OC IV, 574.

50. $L M$ III, in OC IV, 1092. 
l'objectité de la représentation ${ }^{51}$. À cette cécité native, s'oppose le rapport tout aussi natif à l'étant que déploie notre sensibilité, notre être sensible ${ }^{52}$.

En un deuxième moment, la disqualification des facultés cognitives n'atteint pas seulement les sens, mais aussi l'entendement, et, avec les Éléments d'Euclide, ce qui passe pour sa plus parfaite réalisation ${ }^{53}$.

C'est en un troisième temps seulement que Rousseau fait intervenir Descartes dont l'échec est pour ainsi dire emblématique de l'insuccès de la philosophie tout entière, ou, pour le moins, d'une philosophie de l'entendement. Pour le montrer, la lettre s'engage, à l'intention de Sophie, dans un bref exposé de la métaphysique cartésienne (ou du moins de ses démarches initiales), qu'il nous faut maintenant relire:

Avec quelle défiance devons nous nous livrer à nos foibles lumiéres, quand nous voyons le plus methodique des Philosophes, celui qui a le mieux établi ses principes et le plus consequemment raisonné, s'égarer dès les prémiers pas et s'enfoncer d'erreurs en erreurs dans des sistêmes absurdes. Descartes voulant couper tout d'un coup la racine de tous les préjugés commença par tout révoquer en doute, tout soumettre à l'examen de la raison; partant de ce principe unique et incontestable: je pense, donc j'existe, et marchant avec les plus grandes précautions, il crut aller à la vérité et ne trouva que des mensonges. Sur ce premier principe il commença par s'éxaminer, puis trouvant en lui des proprietés très distinctes et qui sembloient appartenir à deux différentes substances, il s'appliqua d'abord à bien connoitre ces deux substances et écartant tout ce qui n'étoit pas clairement et necessairement contenu dans leur idée, il definit l'une la substance étendue, et l'autre la substance qui pense. Définitions d'autant plus sages qu'elles laissoient en quelque sorte l'obscure question des deux substances indécise, et qu'il ne s'ensuivoit pas absolument que l'étendue et la pensée ne se pussent unir et pénétrer dans une même substance. Hébien, ces definitions qui sembloient incontestables furent detruites en moins d'une génération. Newton fit voir que l'essence de la matiere ne consiste point dans l'étendue, Locke fit voir que l'essence de l'ame ne consiste point dans la pensée. Adieu toute la philosophie du sage et methodique Descartes ${ }^{54}$.

51. "Chacun de nous n'appercevant aucun objet se fait de tous une image fantastique», LM III, in OC IV, 1092, nous soulignons. Voir encore la déréalisation du monde de la vue (ibid., p. 1098-1099), qui n'atteint que des spectres légers, et de vains fantômes, c'est-à-dire, précisément, des non-êtres. On comprend que, dans cette destitution du prestige de la vue, Malebranche ait pu fournir un utile réservoir d'arguments tout prêts.

52. Si nous sommes aveugles nés, «nous naissons sensibles» cependant, pour parler comme le début de l'Émile, liv. I, in OC IV, 248. La sensibilité se déploie donc à un tout autre niveau épistémique, mais aussi ontologique, que la vision et la visibilité.

53. LM III, in OC IV, 1095.

54. Ibid., p. 1095-1096. 
Arrêtons-nous un instant sur cet exposé, évidemment très partiel, dont les choix sont en soi significatifs.

1) Selon un topos courant dès les premières heures de la réception du cartésianisme, Descartes est considéré comme le plus méthodique des philosophes. Il est, en tout sens, et pour Rousseau encore, un philosophe exceptionnel. Son échec n'en sera, en dernière analyse, que plus symptomatique de l'impuissance d'une raison laissée à elle-même, ou des prétentions de la connaissance objective.

2) Le «je pense donc j'existe» est bien une vérité première; ainsi qu'il le fera encore dans la Profession de foi, Rousseau lui maintient son caractère unique et incontestable. Il reste toutefois à déterminer en quoi cet énoncé conserve une pertinence et une fonction principielle ${ }^{55}$, ce que fera la Profession. En revanche, le lien de cette affirmation initiale avec le doute qui lui permettait d'émerger est coupé ${ }^{56}$.

3) Conformément à la lettre du cartésianisme («ego sum ego existo»), le «je suis» est spontanément formulé en termes existentiels. Jean-Jacques confirme ainsi que l'existence constitue désormais le sens privilégié de l'être, signification qui a supplanté l'essence et les autres déterminations catégoriales. La certitude première est donc celle d'un existant, qui est le je.

4) «Sur ce premier principe il commença par s'examiner». Autrement dit, l'assertion «j'existe» (qui est un premier principe) ne pose pas simplement une existence plus certaine; il offre le modèle d'une vérité assurée et parfaite, en ce qu'elle porte en elle-même la marque de sa vérité. La formule «sur ce principe» signifie donc tout d'abord qu'en prenant cette vérité pour modèle, et procédant à partir d'elle, nous recherchons ce que nous pouvons dire

55. On peut distinguer, comme le fait L. Verhaeghe, le principe (bien établi par Descartes) et les «premiers pas» qui, d'emblée, nous fourvoient. Si les «premiers pas» renvoient à l'évidence aux vérités générales de la physique, ce qui permet d’épargner la métaphysique ( $\mathrm{Sa}$ physique est d'autant plus touchée que ses principes sont épargnés », écrit l'auteur précité), on peut aussi considérer que les «premiers pas» concernent les vérités de métaphysique immédiatement déduites du principe incontestable. Prêtons attention à l'enchaînement des propositions: "partant de ce principe unique et incontestable: je pense, donc j'existe, et marchant avec les plus grandes précautions, il crut aller à la vérité et ne trouva que des mensonges» (LM III, in OC IV, 1095). Cette première séquence peut sembler passer immédiatement de la métaphysique à la physique (les mensonges). Mais relisons la suite: «Sur ce premier principe [le je pense] il commença par s'éxaminer puis trouvant en lui des proprietés très distinctes et qui sembloient appartenir à deux différentes substances [...]»; entendons: du premier principe que constitue le cogito, il commença à déduire d'autres vérités (toujours métaphysiques); les "premiers pas", alors, désignent la suite de la métaphysique, probablement fausse, puisque même la distinction des substances est relativisée dès les lignes suivantes.

56. Cette disparition est ici d'autant plus frappante que le doute n'est pas absent du début de l'exposé des Lettres morales. 
de nous-mêmes, et comment nous pouvons en être assurés. «J'existe», qui procède de ou s'identifie à «je pense », est une vérité paradigmatique, en tant qu'elle vérifie exemplairement les critères de vérité antérieurement définis. Mais l'expression «sur ce principe» signifie en outre que notre existence constitue la base, ou le matériau phénoménologique de l'investigation à conduire. En d'autres termes, c'est à même et à partir du "j'existe», correctement interprété, qu'il faudra puiser de quoi reconstruire la philosophie. C'est précisément ce à quoi s'attachera le vicaire ${ }^{57}$. L'existence s'affirme avant toute essence. Elle n'est pas la constatation vide d'un fait pur et simple, mais recèle toutes les richesses d'un vivre qui se concentre dans une expérience et un sentiment susceptible d'une intensité indéfiniment variable.

5) L'acuité de la lecture rousseauiste se confirme avec le discernement de deux plans, s'agissant de la distinction réelle des substances. Conscient de l'écart entre distinction au niveau des deux idées («Méditation seconde») et distinction substantielle («Méditation quatrième»), Rousseau rend compte de la définition des deux substances par l'hétérogénéité manifestée dans leurs idées. Au niveau de la seule philosophie rationnelle dont il est ici question, et pour maintenir, stratégiquement, la visée suspensive de son propos, il réserve donc la possibilité d'un monisme réel, alors même qu'il affirme la distinction eidétique: la pensée et l'étendue peuvent n'être en fait qu'une seule et même substance, et la matière pourrait donc penser, selon l'hypothèse d'origine lockienne ${ }^{58}$, âprement discutée à l'époque. Il admet toutefois la pertinence partielle de la distinction cartésienne. En un mot, Rousseau comprend parfaitement la différence entre la «Méditation seconde» et la «Méditation sixième». C'est en ce point que Jean-Jacques interrompt son examen du cartésianisme, comme si, à l'instar de Husserl plus tard, il ne convenait pas d'aller au-delà de la «Méditation seconde».

De ce bref parcours cartésien, on peut tirer deux conséquences.

Tout d'abord, de manière anecdotique, Rousseau laisse à l'histoire le soin de juger, Locke et Newton ayant chacun fait justice des prétentions de la raison à saisir des essences. Mais cette double récusation a pour lui valeur d'indice, et confirme l'insuffisance de la raison à poursuivre au-delà d'une affirmation principielle.

57. À la question «qu'est-ce qui existe ?» (OC IV, 1099), formulée dans le finale de la $L M$ III, répond, quelques lignes plus bas, le «j'existe» seul assuré. En ce sens, avec une rigueur et une incomparable profondeur, Rousseau accomplit la concentration de l'existence sur l'ego, qui caractérise selon nous l'aboutissement du mouvement initié par l'invention cartésienne de l'ego existo. Nous nous permettons de renvoyer ici aux chapitres terminaux de notre Penser l'existence, t. II (époque moderne), Paris, Vrin, à paraître.

58. Voir Locke, Essai, IV, ch. iii, $\$ 6$. La Profession de foi tranchera la question, en optant, dans la perspective de sa réfutation du matérialisme, pour la négative, Émile, liv. IV, in OC IV, 584. 
En second lieu, seul le contenu des deux premières méditations doit nous retenir. Les deux pages qui suivent, dans la troisième Lettre morale, multiplient les fins de non-recevoir opposables aux principales connaissances que Descartes entendait fonder en raison: «Il crut aller à la vérité et ne trouva que des mensonges " ${ }^{59}$. Il nous faut donc comprendre que la vérité du cartésianisme est partielle: ne serait à retenir que l'acquis fondamental des deux premières méditations, le je pense, ou plutôt le j'existe, conformément à la formule reprise à l'entame de la Profession de foi. La métaphysique cartésienne ne vaudrait que par et dans son moment inaugural, c'est-à-dire dans l'affirmation indubitable d'une existence dont tout l'être est de penser, ou plutôt de sentir, comme on le verra bientôt. À la puérilité des philosophes qui veulent pénétrer les essences des choses ou percer le système de l'univers avec un entendement qui n'est pas fait pour cela, on opposera la sagesse de Descartes qui a mis au jour l'essence du premier principe, même s'il n'a pas su s'arrêter à temps. Il nous faut donc «finir par où Descartes avait commencé. Je pense, donc j'existe» ${ }^{60}$.

Cette affirmation déroutante suscite immédiatement plusieurs questions, d'autant plus urgentes qu'elle semble, à la lettre, contradictoire, et immédiatement démentie par les faits, puisque l'exposé des Lettres morales se poursuit, bien au-delà de cette affirmation sensée clore la méditation philosophique.

Quel sens faut-il donner à ce propos, si on entend le concilier avec le fait que Rousseau a développé, avant et après ses Lettres morales, une philosophie entière? Les principes dégagés dans les deux Discours, ou dans la lettre à Voltaire d'août 1756 sont-ils frappés d'incertitude ou de nullité par ce retour au seul «je pense»? Il est inutile d'insister dans cette voie, puisqu'il est entendu que Rousseau n'a jamais opéré une telle remise en cause.

\section{La polysémie du finir}

On peut proposer l'hypothèse suivante: «il nous faut finir», mais il est plusieurs manières de finir. On en distinguera trois, possibles et plausibles, à partir de ce que nous dit la troisième Lettre morale.

1) À l'évidence, le «finir» de Rousseau ne signifie pas un achèvement volontaire, ou la nécessité de se taire, pour s'enfermer dans la pure et simple autoposition d'un «je pense» sans contenu.

59. La fin de non-recevoir est suffisamment vague pour permettre qu'on la fasse porter conjointement sur la métaphysique (preuve de l'existence de Dieu ou du monde extérieur par exemple), ou sur les fondements de la physique (matière subtile, tourbillons, etc.).

60. LM III, in OC IV, 1099. On note que ce passage vise autant Descartes que les philosophes postérieurs, et stigmatise l'orgueilleuse prétention des métaphysiciens à connaître l'essence du réel et le fond des substances. 
2) Dans notre texte, «finir» signifie d'abord achever le parcours du vain savoir. Il faut finir, au sens d'en finir avec les incertitudes sans fin de la philosophie, dont on vient d'étaler complaisamment les faillites. Il faut en finir avec la raison des raisonneurs, pour en venir à autre chose, comme le fera la quatrième Lettre morale, et surtout la Profession de foi. Finir, ce n'est pas achever, mais créer les conditions d'un nouveau commencement ${ }^{61}$. En effet, la Profession semble bien faire de cette fin proclamée, la condition d'un nouveau départ. Et de fait, c'est par un nouveau «j'existe» que s'inaugure l'exposé du vicaire.

3) Dans cette perspective, il apparaîtra que, d'un auteur à l'autre, le «je pense» lui-même change de contenu. Il semble du moins que Rousseau privilégie l'un des modes de la pensée énumérés par Descartes. On pourrait alors finir par l'énoncé cartésien, parce que l'assertion du principe «j'existe» reçoit une signification nouvelle, irréductible à la simple présence à soi de la pensée. Autrement dit, c'est parce qu'on peut donner à "finir» un autre sens que celui d'un achèvement, qu'on peut aussi donner à «je pense» un autre sens que celui d'un commencement. Le «je pense donc j'existe» serait «final», parce qu'il récapitulerait en lui les conditions de l'expérience du rapport à soi-même, aux autres hommes, et, en définitive, à tout ce qui est. Pour l'entendre, il ne faudra pas s'en tenir à l'exposé de la troisième Lettre morale. La Profession de foi se livre en effet à une véritable reformulation du premier principe. On pourra alors, sans trop jouer sur les mots, commencer par où Descartes a fini. Entendons par là que la reformulation rousseauiste privilégie le mode ultime de la pensée, le sentir, qui, Descartes l'a montré et Rousseau le confirme à sa manière, implique la co-présence du corps et de l'esprit.

\section{Une réécriture du cogito}

Venons-en maintenant à la Profession de foi, à propos de laquelle on fera l'hypothèse suivante, qui s'énonce en deux temps.

61. Relisons le final de la $L M$ III (in OC IV, 1099) : « Après avoir parcouru le cercle étroit de leur vain savoir, il faut finir par où Descartes avoit commencé. Je pense, donc j'existe. Voilà tout ce que nous savons». C'est là tout ce que nous savons (et qui s'excepte ainsi du vain savoir dont on vient de prendre congé), mais cela suffit pour la relance à venir de la philosophie dans l'exposé du vicaire. Quelles que soient la manière d'appréhender l'épistémologie du vicaire et l'importance qu'on doit accorder au thème des limites de l'entendement dans la Profession de foi, reste que le vicaire tient en partie pour vraies des assertions rejetées dans les Lettres à Sophie. En témoigne, par exemple, la réhabilitation (au moins relative) d'une notion de substance (Émile, liv. IV, in OC IV, 576, et surtout 584), totalement évincée par la $L M$ III, in OC IV, 1099. 
1) L'exposé du vicaire peut se lire comme la mise en œuvre d'un cogito. Le début de la Profession (on entend par là les trois premiers articles de foi) ${ }^{62}$ peut se comprendre comme le déploiement des vérités qui nous importent à partir d'un premier principe («j'existe et j'ai des sens»), mais aussi comme l'exploration pour ainsi dire phénoménologique des différents modes du penser ainsi retravaillé. Le geste initial du vicaire inscrit la subjectivité au cœur de la pensée, et en accuse ainsi la centralité.

2) Ce cogito ne peut toutefois se réduire à sa littéralité cartésienne, et n'autorise pas les résultats qu'il rendait possible dans le cartésianisme. Il n'est donc pas une répétition. Il ne conduit pas à l'explicitation de la nature de l'âme, sa substance, même si on accède à l'effectivité de ses modes ou propriétés ${ }^{63}$. Il se confirmera donc que, parmi les acquis cartésiens, Rousseau sélectionne et transpose, en vue de son propre projet.

Pour des raisons de clarté, commençons par le second point. Deux motifs interdisent l'hypothèse d'une simple répétition rousseauiste du cogito cartésien, en dépit de l'analogie de situation que Jean-Jacques mentionne $(O C I V, 567)$, en faisant dire à son vicaire qu'il se trouve dans les dispositions d'incertitude et de doute que Descartes exige pour la recherche de la vérité. Un premier motif concerne le doute, un second, le «je pense».

En premier lieu, les conditions dans lesquelles émerge le principe, ne satisfont pas aux exigences cartésiennes. Le «je pense» ne résulte pas d'un doute naturel, et encore moins hyperbolique. À doute différent (en sa nature comme en ses objets), cogito différent. Si on peut considérer que nos deux auteurs s'accorderaient pour reconnaître que le doute est un état violent et peu fait pour durer, l'analogie s'arrête là. Comme l'a bien vu Henri Gouhier (qui n'en tire peut-être pas toutes les conséquences), le doute du vicaire est existentiel (au sens actuel du terme), et non volontaire ${ }^{64}$. Il porte en priorité sur le corps des vérités morales et religieuses, que Descartes laissait précisément hors du doute ${ }^{65}$. En écho à la troisième Lettre morale,

62. Rappelons-les pour mémoire: 1) il existe une volonté motrice de l'univers et animatrice de la nature (Émile, liv. IV, in OC IV, 576); 2) une intelligence ordonnatrice et législatrice (ibid., p. 578);3) l'homme est libre et doté d'une âme immatérielle (ibid., p. 586-587); «De ces trois premiers vous déduirez aisément tous les autres [...]», ibid.

63. «La volonté m'est connüe par ses actes, non par sa nature» (ibid., p. 576); voir ibid., p. 590. Significative est à cet égard la manière dont le vicaire traite du dualisme substantiel en l'homme: celui-ci n'est pas démontré a priori, comme une thèse de nature proprement métaphysique, mais s'impose, de fait, comme l'explication la plus plausible et la plus économique des combats, voire des contradictions qui traversent le psychisme humain (Émile, liv. IV, in OC IV, 583).

64. Sur le caractère volontaire du doute cartésien, voir Principia philosophice, Pars prima, $\$ 6$, AT VIII-1, 6, et, notamment, H. Gouhier, La pensée métaphysique de Descartes, 4 éd., Paris, Vrin (Bibliothèque d'histoire de la philosophie), 1987, p. 113 sq.

65. Voir Principia philosophice, Pars prima, $\$ 3$, AT VIII-1, 5 et $\$ 25$, p. 14 par exemple. 
le doute du vicaire se résume à constater, pour en finir avec eux, l'incertitude foncière des philosophes. Ce que l'on peut nommer le scepticisme du vicaire n'est donc pas celui des philosophes: le vicaire est sceptique par l'indifférenciation de toutes leurs opinions, qu'il réduit au dénominateur de leur commune incertitude ${ }^{66}$. Plusieurs déplacements modifient donc profondément le rapport du doute au «je pense».

Tout d'abord, la règle de vérité, qui érige le sentiment en opérateur d'évidence ${ }^{67}$, est énoncée avant le «je pense», ou ce qui lui est substitué. La règle de vérité, ou son analogon rousseauiste ${ }^{68}$, n'est pas tirée du «je pense», mais le conditionne. Ensuite, le «premier doute», quant à la teneur (sensorielle ou non) du sentiment d'existence, est énoncé après le premier principe ${ }^{69}$. Ce doute est dit "premier», ce qui laisse deviner qu'il diffère de ceux qui précèdent l'énoncé du «j'existe». Il est le premier, au sens des doutes spéculatifs ou théoriques; il se distingue ainsi des doutes pour ainsi dire axiologiques qui animaient et habitaient les pages précédentes. Il reste (et ceci est notoire) que ce doute porte sur la nature du sentiment d'existence, autrement dit sur ce que la première vérité est censée exposer en pleine lumière ${ }^{70}$. Le «j'existe»

66. Immédiatement après avoir évoqué ses lectures aux Charmettes, Jean-Jacques ajoute: «Je m'apperçus bientot que tous ces auteurs étoient entre eux en contradiction presque perpétuelle, et je formai le chimérique projet de les accorder, qui me fatigua beaucoup et me fit perdre bien du tems", Confessions, liv. VI, in OC I, 237.

67. OC IV, 570 .

68. Il serait plus exact d'évoquer la règle d'évidence, comme si ce qui est évident (ou découle de principes évidents) devait ipso facto être tenu pour vrai. Concernant la réécriture rousseauiste de la règle et les déplacements que Jean-Jacques lui impose, voir A. Charrak, «Descartes et Rousseau ", in Rousseau et la philosophie (Actes du colloque de Paris, 11-12 mai 2001), A. Charrak, J. Salem (dir.), Paris, Publ. de la Sorbonne, 2004, p. 23, repris et développé dans A. Charrak, Rousseau de l'empirisme à l'expérience, Paris, Vrin (Bibliothèque d'histoire de la philosophie), 2012, p. 202-204. En revanche, il ne nous semble pas nécessaire de recourir à une médiation condillacienne dans la réception rousseauiste de la règle malebranchiste dont on trouve l'énoncé canonique en Recherche de la vérité, I, II, $₫$ iv, Les Confessions, liv. II, in OC I, 55. Le Traité des systèmes, chap. VI (F. Markovits, M. Authier (éd.), Paris, Fayard, 1991, p. 63) évoque bien la règle de l'évidence, mais en des termes qui nous paraissent bien plutôt s'éloigner à la fois du «patron» malebranchien et de la reprise rousseauiste. Un glissement est tout spécialement notable: alors que Malebranche voit encore dans la raison (et non ici dans le sentiment intérieur) et les reproches secrets qu'elle nous peut faire l'instance de ratification de l'évidence authentique, Condillac transfère ce pouvoir à la conscience. La présence, dans les deux textes, de la notion de reproches secrets ne nous semble pas légitimer le rapprochement, tant peut s'avérer grande la distance entre raison et conscience. Ajoutons que ce rôle dévolu par Condillac à la conscience ne nous semble pas malebranchiste, comme le suggère A. Charrak, dans Rousseau de l'empirisme à l'expérience, p. 204.

69. OC IV, 570-571.

70. «Ai-je un sentiment propre de mon existence, ou ne la sens-je que par mes sensations? Voilà mon prémier doute, qu'il m'est, quant à présent, impossible de résoudre ", Émile, liv. IV, in OC IV, 570-571. 
rousseauiste est donc en déficience sur le «je pense» cartésien, puisqu’il ne livre plus accès à la nature de ce qui se donne à sentir.

Un second motif interdit d'évoquer une réitération du cogito cartésien. Rousseau refuse en effet une thèse cartésienne fondamentale (inscrite au titre même de la "Seconde méditation»), lorsqu'il affirme que nous ne connaissons pas la nature de notre âme. La pensée, dans sa réflexivité, ne peut appréhender sa propre essence: «Je sens mon ame; je la connois par le sentiment et par la pensée; je sais qu'elle est, sans savoir quelle est son essence; je ne puis raisonner sur des idées que je n'ai pas ${ }^{71}$. On notera l'impossibilité d'un retour au cartésianisme orthodoxe, tant est ici prégnante sa subversion malebranchiste: l'âme se connaît non pas seulement par le sentiment (Malebranche) mais bien par la pensée (Descartes), et pourtant n'accède pas à sa nature (Malebranche de nouveau, et une bonne part du XVIII ${ }^{e}$ siècle à sa suite). Avec la réflexion et la représentation, deux modèles de la connaissance de soi sont d'emblée disqualifiés. La réflexion tout d'abord, ce qu'atteste la « dissertation sur le jugement ${ }^{72}$, pour parler comme Pierre-Maurice Masson ${ }^{73}$. La réflexion apparaît, en équivalence avec l'attention ou la méditation (une telle indétermination est déjà significative) pour rendre compte de notre pouvoir de comparer les sensations, et finalement de porter des jugements. Si la réflexion est bien une fonction active de l'esprit, elle ne porte plus en priorité sur la connaissance de soi. La représentation, quant à elle, avait déjà été exclue par la troisième Lettre morale qui nous refusait la vision de notre âme parce que nous n'avons pas de miroir intellectuel qui nous la montre, ajoutait Rousseau, avant de biffer ces derniers mots ${ }^{74}$. Quoi qu'il en soit, et quelque sens qu'on donne à l'image du miroir, la signification du refus est claire: l'âme, et la pensée qui continue d'en constituer l'essence, est inobjectivable ${ }^{75}$.

On peut dès lors comprendre que Rousseau ait éludé (par sa réécriture même) le «je pense» dans l'exposé publié de la Profession de foi. Ce qui lui importe d'abord, c'est de s'assurer de soi, selon sa propre expression,

71. OC IV, 590.

72. OC IV, 573.

73. Voir J.-J. Rousseau, La "Profession de foi du vicaire Savoyard”, P.-M. Masson (éd.), Fribourg, O. Gschwend (Collectanea Friburgensia; 25), 1914.

74. OC IV, 1092.

75. Le vocabulaire de la représentation est, sauf erreur, absent de la Profession de foi, hormis l'évocation polémique des "sensations représentatives" (Émile, liv. IV, in OC IV, 572) qui renvoie probablement à l'article «Évidence» de l'Encyclopédie (éd. Masson, p. 85, $\mathrm{n}^{\mathrm{o}} 2$ ). Voir Émile, liv. I, in OC IV, 282. On ne peut ici développer l'influence de l'article «Évidence» sur les vues rousseauistes en matière de théorie de la connaissance. Dans notre présente perspective, on sera cependant attentif à la notion de sensation affective, et son rôle déterminant jusque dans les connaissances proprement objectives. 
c'est-à-dire d'affirmer la certitude de son existence comme être affecté: "J'existe et j'ai des sens par lesquels je suis affecté» ${ }^{76}$. Il n'est donc pas requis que la pensée se pense soi-même pour produire cette certitude. Significatif est à cet égard le fait, déjà rappelé, que le sentiment qui opère la certitude première de notre existence, et qui nous délivre ce que nous appelons moi, soit d'étoffe sensible, sinon sensorielle. La certitude première, qui est celle de notre existence, ne requiert pas la suspension expresse et volontaire des objets du monde, même si les premières pages de la Profession, dans une subtile reprise des éléments de la troisième Lettre morale, exhibe un mode de notre rapport à l'être qui dépasse la représentation.

À la lumière de ces quelques rappels, on ne saurait conclure à une répétition du cogito sous sa forme cartésienne ${ }^{77}$. Mais si Rousseau ne répète pas Descartes, ignore-t-il pour autant ce qu'un «je pense» rend possible?

\section{Du «je pense» au «je suis affecté»}

Nous pouvons maintenant revenir au premier moment de notre hypothèse, pour nous demander ce que le vicaire doit au «je pense» et tenter de préciser ce que peut être son cartésianisme.

L'incipit de l'exposé ${ }^{78}$ opère la transposition ou du moins l'infléchissement affectif du cogito, tout en maintenant simultanément son caractère principiel: c'est à partir du «j'existe et j'ai des sens», et à même ce qui se donne en ce sentir originaire, qu'on va progresser vers les vérités qui importent ${ }^{79}$. C'est dire que Rousseau opère un déplacement déterminant, qui décèle une des significations du geste cartésien.

On trouve confirmation de cette centralité métaphysique dans le mouvement de pensée et l'enchaînement des vérités (articles de foi) qui gouverne l'exposé du vicaire.

En préambule, partons de quelques remarques touchant la reformulation rousseauiste de la règle de vérité ${ }^{80}$. Trois points sont à noter.

76. OC IV, 570.

77. On ne peut conclure, à la suite de Henri Gouhier (Les méditations métaphysiques...), et de Paul Audi (Rousseau, une philosophie de l'âme, p. 356), à une «reprise sans tergiversation du cogito cartésien ».

78. OC IV, 570 .

79. La fin de l'exposé proprement métaphysique donne la clé méthodologique de l'ensemble: "Après avoir ainsi de l'impression des objets sensibles et du sentiment intérieur qui me porte à juger des causes selon mes lumiéres naturelles déduit les principales vérités qu'il m'importoit de connoitre, il me reste à chercher quelles maximes j'en dois tirer pour ma conduite [...]», Émile, liv. IV, in OC IV, 594.

80. «Portant donc en moi l'amour de la vérité pour toute philosophie, et pour toute méthode une régle facile et simple qui me dispense de la vaine subtilité des argumens, je reprends 
a) On n'examine que les "connaissances qui m'intéressent», ce qui annonce, quelques lignes plus bas, la réduction de l'enquête aux vérités de pratique. Il est inutile d'insister longuement sur l'anti-cartésianisme de ce déplacement, qui élimine, au moins en apparence, le projet de fonder en métaphysique le corps des autres sciences, et notamment une vaine physique soucieuse de percer la nature des choses ${ }^{81}$.

b) Au rebours de son étymologie, l'évidence n'est plus pensée comme un voir, comme un dévoilement, mais comme un sentir dont la teneur l'emporte précisément sur toute évidence d'entendement ou de représentation $^{82}$. Cette teneur qui se donne comme indubitable et se révèle dans et par l'impossibilité de ne pas consentir se déploie dans la sincérité du cour, ailleurs identifiée au sentiment intérieur. Les pages qui précèdent, en narrant le malaise moral et spirituel du vicaire, ont précipité cette crise de l'évidence; il faut donner tout son poids à une formule brève, qui peut sembler banale: "aucun de nous ne peut voir ce qui est ${ }^{83}$. Au-delà d'un poncif sceptique, il faut comprendre, comme nous y invitait déjà la troisième Lettre morale, que le rapport à ce qui est ne relève pas du voir, mais bien du sentir. Ce que nous avons improprement nommé le scepticisme de la troisième Lettre prend alors tout son sens, en ce qu'il vise d'abord à ébranler le faux prestige de la vue, ou plus exactement la domination injustifiée du paradigme de la visibilité dans la compréhension correcte de notre rapport à ce qui est. Ce qui est, précisément, c'est-à-dire plus exactement ce qui existe, ne se donnera qu'au travers de ce que nous en délivre l'affection par un sentiment ${ }^{84}$.

c) On admet pour vraies toutes les connaissances qui ont une liaison nécessaire avec les premières. Les connaissances subséquentes seront tenues pour vraies dès lors qu'elles s'enchaînent aux précédentes, qui, à leur égard,

sur cette régle l'éxamen des connoissances qui m’intéressent, résolu d'admettre pour évidentes toutes celles auxquelles dans la sincérité de mon cœur je ne pourrai refuser mon consentement, pour vrayes toutes celles qui me paroitront avoir une liaison nécessaire avec ces prémiéres, et de laisser toutes les autres dans l'incertitude, sans les rejetter ni les admettre, et sans me tourmenter à les éclaircir quand elles ne mênent à rien d'utile pour la pratique", Émile, liv. IV, in OC IV, 570.

81. Voir déjà $L M$ III, in OC IV, 1099 (immédiatement avant la condamnation du vain savoir des philosophes): «Nous ne conoissons pas une substance dans l'univers, nous ne sommes pas même surs d'en voir la surface et nous voulons sonder l'abime de la nature. Laissons un si puerile travail à ces enfants qu'on appelle des philosophes ".

82. Voir encore la Profession de foi, Émile, liv. IV, in OC IV, 574. Le lieu de l'évidence n'est plus l'intuition de l'entendement, mais le cœur qui sent, pour paraphraser Pascal (Pensées, fragment Lafuma 424).

83. OC IV, 568.

84. Dès le livre premier, l'Émile développait ainsi le «nous naissons sensibles» déjà cité: «et dès nôtre naissance nous sommes affectés de diverses maniéres par les objets qui nous environnent» (Émile, liv. I, in OC IV, 248). 
tiennent le rôle de principe. Une assertion quelconque détient sa vérité et sa certitude de sa connexion et de sa déductibilité à partir d'une vérité sentie antérieurement. On ne peut évidemment s'empêcher de songer à la troisième règle cartésienne de la méthode, et, plus largement, à l'instauration d'un ordre des raisons ${ }^{85}$. Or l'apparition de ce philosophème typique du cartésianisme en ce nouveau contexte n'est pas le fruit du hasard, ou de la simple coquetterie littéraire.

Revenons à la première vérité qui s'énonce au début de l'exposé métaphysique de la Profession, pour en souligner trois aspects.

Dans l'interprétation qu'en donne Rousseau, «j'existe» ne renvoie pas à une simple conscience de soi; il contient déjà l'élucidation de l'être de l'homme comme être sensible. Ce «j'existe» est premier, parce que la sensibilité par laquelle il émerge et s'impose est elle-même originaire: «j'existe, et j'ai des sens par lesquels je suis affecté». Les deux assertions sont absolument conjointes. Je n'existe que comme être affecté, et, serait-on tenté d'ajouter, que dans la stricte mesure où je le suis: «Exister pour nous, c'est sentir ", redira le vicaire un peu plus $\operatorname{tard}^{86}$. La question de savoir si le sentiment d'existence est ou non indépendant des sensations ne contredit en rien l'enracinement de l'être dans le sentir ${ }^{87}$. Le début de la Profession opère la réduction de l'objet extérieur à une idée, et de la matière à ce qui en est senti. De ce fait, le vicaire peut s'inscrire en deçà du débat entre idéalistes et matérialistes, précisément au titre de cette réduction au sentir ${ }^{88}$.

85. «Le troisieme [précepte], de conduire par ordre mes pensées, en commençant par les objets les plus simples et les plus aysez a connoistre, pour monter peu à peu, comme par degrez, jusques a la connoissance des plus composez», Discours de la méthode, AT VI, 18.

86. Émile, liv. IV, in $O C$ IV, 6oo. Il faudrait en outre analyser en détail ces passages de $L a$ Nouvelle Héloïse: «Je n'ai jamais mieux remarqué avec quel instinct je place en divers lieux notre existence commune selon l'état de mon ame. Quand je suis triste, elle se réfugie auprès de la votre, et cherche des consolations aux lieux où vous êtes; c'est ce que j'éprouvois en vous quitant. Quand j'ai du plaisir, je n'en saurois jouir seul, et pour le partager avec vous, je vous appelle alors où je suis », première partie, XXIII, in OC II, 83 ; «Si je ne t'aime plus? Quel doute! ai-je donc cessé d'exister, et ma vie n'est-elle pas plus dans ton cœur que dans le mien? Je sens, je sens que tu m'es mille fois plus chere que jamais, et j'ai trouvé dans mon abatement de nouvelles forces pour te chérir plus tendrement encore», première partie, LV, in OC II, 149; « Je sentois que j'allois perdre avec la moitié de toi-même une partie de ma propre existence. Je comptois les heures avec inquietude, et voyant poindre le jour, je n'ai pas vu naître sans effroi celui qui devoit décider de ton sort", première partie, LXV, in $O C$ II, 182.

87. S'agissant de l'équivalence de l'être et du sentir, voir la lettre à Jacob Vernes du 18 février 1758: "Cela me fait soupçonner qu'il se pourroit bien que les ames des méchans fussent anéanties à leur mort et qu'étre et sentir fut le premier prix d'une bonne vie», Lettres philosophiques, H. Gouhier (éd.), p. 54; «et puisqu'ils [les matérialistes] sont surs qu'ils ne pensent pas, comment osent-ils affirmer qu'ils sentent? ", Profession de foi, in Émile, liv. IV, in OC IV, 585 , note de Rousseau.

88. Ibid., p. 571. 
Ajoutons que, dans les deux grands textes des Rêveries (promenades II et V) ${ }^{89}$ relatifs au sentiment d'existence, celui-ci suppose l'affaiblissement des sensations externes, non pas leur totale suspension. Le je se trouve donc institué comme principe, et l'affection comme fondement de ce qui va se dire. Nous naissons sensibles, lisons-nous au début de l'Émile ${ }^{90}$. La sensibilité n'est pas un acquis, mais nous est éminemment naturelle, ce qui ne lui interdit pas d'être éduquée. Elle est pour l'homme, sa manière fondamentale d'être au monde ${ }^{91}$. L'élision du «je pense» libère la possibilité de ne plus comprendre le je existant comme un sujet de la représentation, mais de revendiquer pour lui le primat de l'apparaître. Nous avons bien lu: «j'ai des sens par lesquels je suis affecté». Le "par» admet (ou du moins tolère) deux lectures: une première, pour ainsi dire passive, fait du sens le simple canal, par lequel les choses mêmes nous affectent; une autre interprétation, active peut-on dire, pense le sens (et non plus la chose même) comme la source de l'affection. Ce ne sont plus les choses qui affectent, mais les sens eux-mêmes. Le «j'existe», comme être affecté, se leste d'une signification proprement ontologique.

Le «j'existe» est, par ailleurs, une première vérité, d'où jaillit immédiatement l'existence du monde, aussi certaine que la nôtre, puisqu'elle s'en tient à l'épreuve de diverses sensations. Or leur caractère éprouvé confirme que le moi ne peut en être la cause. Convient-il, pour conférer tout son poids à cette primauté du sensible, d'évoquer un «cogito sensible ou affectif»? Une dernière remarque pourrait nous y conduire. Ne nous méprenons pas sur la signification des premières pages de l'exposé: ce qu'on y lit, et notamment que nous existons et avons des sens, n'est pas si rebattu que le pense Voltaire ${ }^{92}$. Contre le sensualisme des philosophes, et notamment contre la doctrine dite de la sensation transformée (d'origine condillacienne), l'effort de Rousseau consiste à mettre en évidence la spontanéité de la pensée, à partir du flux des sensations. Les pages relatives à la nature active du jugement, qui s'oppose à la passivité des sensations, sont bien connues, et souvent commentées. On se bornera à une remarque, sur la base du texte consacré à la fonction de comparaison ${ }^{93}$. La capacité

89. Voir Deuxième promenade, in $O C \mathrm{I}$, 1005, et Cinquième promenade, in OC I, 1045-1047 (qu'il faudrait reprendre en tout son détail).

90. Émile, liv. I, in OC IV, 248.

91. Le vicaire évoque ainsi (ici à propos de Dieu) «l'amour de soi essentiel à tout être qui se sent», Émile, liv. IV, in OC IV, 588.

92. Cité par H. Gouhier, Rousseau et Voltaire. Portraits dans deux miroirs, Paris, Vrin (Bibliothèque d'histoire de la philosophie), 1983, p. 186.

93. «Appercevoir, c'est sentir; comparer, c'est juger; juger et sentir ne sont pas la même chose. Par la sensation, les objets s'offrent à moi séparés, isolés, tels qu'ils sont dans la nature; par la comparaison, je les remüe, je les transporte, pour ainsi dire, je les pose l'un sur l'autre 
à comparer, qui nous vaut "l'honneur de penser", se comprend comme une fonction de rassemblement, conjurant l'isolement des étants en leur état naturel. Penser, c'est comparer, c'est-à-dire regrouper ou rassembler. En un mot, la définition de la pensée reste celle d'une perception, d'un percipere, sinon d'une cogitatio. Mais celle-ci est toujours seconde, et ne peut se substituer à la fonction du sentiment.

C'est bien à partir de cet apparaître qu'on pourra, comme va le faire la suite du texte, progresser vers la reconstruction d'un savoir ${ }^{94}$.

\section{Concluons.}

1) Ainsi qu'il en va pour la plupart des philosophes des Lumières, le rapport de Rousseau à Descartes n'est pas homogène. Il est sélectif et différencié. Au sein du cartésianisme, Rousseau opère des choix en fonction de ses intérêts et de sa propre philosophie. Il abandonne la physique entière, y compris ses principes, puisque l'identification de la matière à l'étendue elle-même est en quelque sorte relativisée dans l'exposé du vicaire ${ }^{95}$. De la métaphysique, il congédie une bonne part, de la démonstration de l'existence de Dieu à la preuve de celle des corps. Mais il épargne son premier principe, parce qu'il est incontestable, mais aussi transposable et qu'il fera l'objet d'une reformulation compatible avec une épistémologie radicalement différente.

2) Rousseau ne répète pas Descartes à la manière d'un cartésien plus ou moins fidèle. Sa distance par rapport au cartésianisme lui donne, paradoxalement, de confirmer ce que le cogito a ouvert et permis. Du «je pense», Jean-Jacques retient deux éléments: contre ce qu'il croit être une dérive naturaliste du sensualisme ambiant, il en use pour attester la transcendance ontique du moi, et de la vie subjective au regard de l'univers physique. Mais cette transposition, ou ce nouvel usage de l'ancien principe, ne s'obtient qu'au prix d'un infléchissement originaire du cartésianisme, que Descartes lui-même rend possible en reconnaissant à la pensée une polysémie originaire. C'est en effet à partir du dernier mode de la cogitatio cartésienne, par lequel l'ego s'appréhende comme "chose qui sent", que le «je pense» rousseauiste conserve sa pertinence. Ce qui s'énonçait en dernier est devenu premier. Ce faisant, à l'instar de Malebranche qu'il médita aussi aux Charmettes, Rousseau

pour prononcer sur leur différence ou sur leur similitude, et généralement sur tous leurs rapports. Selon moi, la faculté distinctive de l'être actif ou intelligent est de pouvoir donner un sens à ce mot est", Émile, liv. IV, in OC IV, 571.

94. On tire du j'existe les principales vérités, à commencer par l'existence du monde extérieur, l'autonomie du jugement au regard des sensations, puis le refus de l'automotricité matérielle (pièce essentielle de la polémique antimatérialiste), acquise à partir de l'expérience originaire du mouvement spontané.

95. Voir sur ce point A. Charrak, «Descartes et Rousseau», p. 27-28. 
devait à Descartes le sentiment qu'il opposait au sien, « et la hardiesse de le reprendre».

3) Sans qu'il soit ici possible d'aller plus avant, la réception rousseauiste du cogito, notamment la manière dont il fonctionne dans l'économie de la Profession de foi, permet, voire impose d'ouvrir, au-delà du dossier Descartes, le débat, autrement plus décisif encore, du rapport de Rousseau à la métaphysique.

Jean-Christophe BARDout Université de Rennes I 